\title{
On the Antijamming Performance of the NR-DCSK System
}

\author{
Binh Van Nguyen $(\mathbb{D}$, Hyoyoung Jung $\mathbb{D}$, and Kiseon Kim $(\mathbb{D}$ \\ School of Electrical Engineering and Computer Science, Gwangju Institute of Science and Technology, Gwangju, Republic of Korea \\ Correspondence should be addressed to Kiseon Kim; kskim@gist.ac.kr
}

Received 13 November 2017; Accepted 16 January 2018; Published 11 February 2018

Academic Editor: Pino Caballero-Gil

Copyright (C) 2018 Binh Van Nguyen et al. This is an open access article distributed under the Creative Commons Attribution License, which permits unrestricted use, distribution, and reproduction in any medium, provided the original work is properly cited.

\begin{abstract}
This paper investigates the antijamming performance of the NR-DCSK system. We consider practical jamming environments including broadband jamming (BBJ), partial-time jamming (PTJ), tone jamming (TJ), and sweep jamming (SWJ). We first analytically derived the bit error rates of the system under the BBJ and the PTJ. Our results show that the system performances under these two jamming environments are enhanced as $P$ increases, where $P$ is the parameter of the NR-DCSK modulation scheme denoting the number of times a chaotic sample is repeated. In addition, our results demonstrate that, for the PTJ, the optimal value of the jamming factor is close to zero when the jamming power is small; however, it increases and approaches one as the jamming power enlarges. We then investigate the performance of the system under the TJ and the SWJ via Monte-Carlo simulations. Our simulations show that single-tone jamming causes a more significant performance degradation than multitone jamming. Moreover, we point out that the system performance is significantly degraded when the starting frequency of the sweep jammer is close to the carrier frequency of the transmitted signals, the sweep bandwidth is small, and the sweep time is half of the transmitted bit duration.
\end{abstract}

\section{Introduction}

Chaotic communication (CC) has recently been considered as a promising alternative to the conventional direct sequence spread-spectrum (DS-SS) systems. The basic idea of CC is to replace pseudonoise sequences by chaotic sequences, which can be directly generated by chaotic maps. Among various chaotic maps, logistic has been extensively exploited due to its simplicity and good performance [1]. Chaotic systems are generally categorized as coherent and noncoherent chaotic systems. In the former systems, chaotic synchronization plays a vital role in deciding the systems performance. However, designing an efficient chaotic synchronization scheme still remains as a challenging problem. As a result, research works on the coherent chaotic systems are very limited. On the other hand, in the latter systems, data recovery procedure is much simpler than that of the coherent counterparts since the chaotic synchronization is not required. Consequently, a lot of efforts have been devoted to design effective noncoherent modulation schemes and to find novel applications of nonchaotic coherent systems in reality [2].

The fundamental and most studied noncoherent modulation scheme is differential chaotic shift keying (DCSK).
In a chaotic system with the DCSK scheme, referred to as DCSK system, a bit duration is divided into two equal time slots. The first slot is for transmitting a chaotic signal, namely, reference signal, which contains $\beta$ different chaotic samples. In addition, depending on the bit to be sent, the reference signal is either repeated or multiplied by a factor of -1 and transmitted in the second slot. At the receiver, the received signal is correlated with its "half a bit"-delayed version to estimate the transmitted information bit. The performances of the DCSK system over the additive white Gaussian noise (AWGN) and the multipath fading channels are extensively investigated in [3], [4], and [5], respectively. It is shown that enlarging the spreading factor beyond a certain value degrades the performance of the DCSK system.

The DCSK system is simple and largely considered; however, it suffers from several drawbacks, that is, low data rate, high energy consumption, using complex wideband delay, and so on. Motivated by this fact, several advanced systems have been recently proposed including reference modulated DCSK [6], high efficiency DCSK [7], improved DCSK [8], short reference DCSK [9], and noise reduction DCSK (NRDCSK) [10], among which the NR-DCSK system provides the best noisy performance. The generation of the reference 
signal in the NR-DCSK system is totally different from that in the DCSK counterpart. Particularly, only $\beta / P$ different chaotic samples are generated and each sample is repeated $P$ times to make a reference signal of $\beta$ samples in total. Due to its advantages, noncoherent chaotic communication has also been applied to other practical systems; that is, DCSK is combined with the index modulation technique to form spatially modulated DCSK and permutation index DCSK systems, DCSK is considered for power line communications and OFDM systems, and the SR-DCSK scheme is recently investigated for simultaneous wireless information and power transfer systems.

Although chaotic systems in general and noncoherent chaotic systems in particular are partly designed for the antijamming (AJ) purpose, the AJ performance of a noncoherent chaotic system is only carried out in [11]. Particularly, the performance of the DCSK system under the single-tone jamming (STJ) environment is studied. The authors derived the system bit-error-rate (BER) in a closed-form expression and showed that the system performance is significantly degraded when the jamming frequency is an integer multiple of the bit frequency. Despite the fact that the fundamental DCSK system is already investigated, the AJ performances of its recent advanced variants have not been reported in the literature yet. To fill this gap, in this paper, we will consider the recent proposed NR-DCSK system and analyse its AJ performance under various practical jamming environments such as broadband jamming (BBJ), tone jamming (TJ), partialtime jamming (PTJ), and sweep jamming (SWJ). We first analytically derived the BERs of the NR-DCKS system under the $\mathrm{BBJ}$ and PTJ in closed-form expressions. We then investigate the BER of the considered system under the TJ and the SWJ via Monte-Carlo simulations. Our analytical and simulation results reveal several novel insights about the effects of the jamming parameters on the system performance.

The remainder of this paper is organized as follows. Section 2 introduces the system model. Practical jamming signals are described in Section 3. In Section 4, we present mathematical frameworks to derive the system BERs under the BBJ and the PTJ environments. Thereafter, representative simulations are provided in Section 5 to verify our analysis and further demonstrate the effects of the TJ and the SWJ on the performance of the considered system, followed by our conclusion in Section 6.

\section{System Model}

The considered system consists of a pair of source-destination and a jammer, as illustrated in Figure 1. Each node has a single antenna. In addition, the NR-DCSK scheme is employed for modulating and demodulating legitimate signals. Moreover, the logistic map is exploited for generating chaotic sequences. Here, the logistic map is considered because of its simplicity and good performance. A mathematical representation of the map is given by [1]

$$
x_{k+1}=1-2 x_{k}^{2},
$$

where $E\left[x_{k}\right]=0, E\left[x_{k}^{2}\right]=1$, and $\operatorname{var}\left[x_{k}^{2}\right]=1 / 2$ for normalized $x_{k}$.

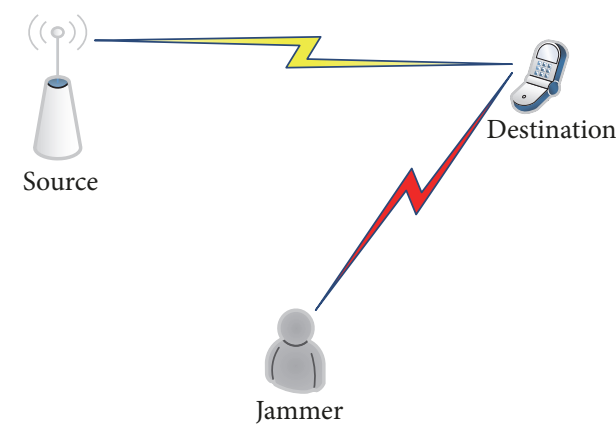

FIGURE 1: NR-DCSK system in a jamming environment.

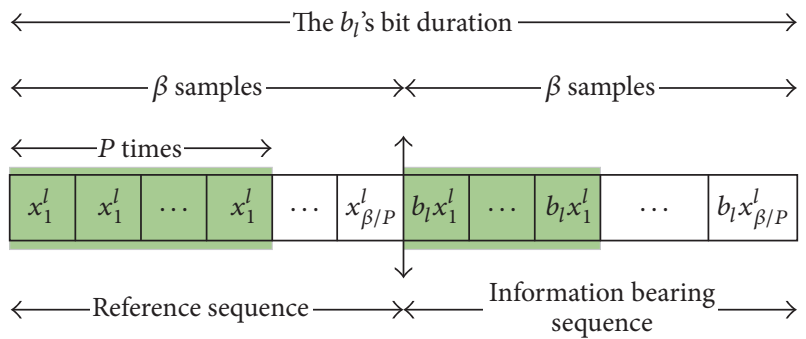

FIGURE 2: A graphical representation of the transmitted signal of the bit $b_{l}$.

For the modulation process, each bit duration is divided into two equal time slots. The first time slot is allocated for transmitting a reference chaotic sequence length $\beta$, called spreading factor, while the second one is used for transmitting either the reference sequence (when the bit being sent is +1 ) or its inverted version (when the bit being sent is -1 ). The reference sequence is generated as follows. The chaotic generator first generates $\beta / P$ samples. Then, each chaotic sample is replicated $P$ times to obtain the reference sequence with length $\beta$ in total. As a result the transmitted signal of the $l$ th bit, $b_{l}$, can be expressed as [10]

$$
s_{k}^{l}= \begin{cases}x_{\lceil k / P\rceil}^{l}, & \text { if } 0<k \leq \beta, \\ b_{l} x_{\lceil k / P\rceil-\beta}^{l}, & \text { if } \beta<k \leq 2 \beta,\end{cases}
$$

where $\lceil\cdot\rceil$ denotes the ceiling operator. Graphically, $s_{k}^{l}$ can be illustrated as in Figure 2.

The received baseband signal at the receiver can be expressed as follows:

$$
r_{k}^{l}=s_{k}^{l}+j_{k}^{l}+n_{k}^{l}
$$

where $j_{k}^{l}$ and $n_{k}^{l}$ are the jamming signal and the AWGN. Here we consider the AWGN channels so that we can solely focus on the effect of jamming on the system performance. The assumption of the AWGN channels has also been adopted in several related works, that is, $[11,12]$. The joint effects of jamming and fading channels on the performance of the NRDCKS system will be considered in our future works. The received signal is first averaged using a block average filter (BAF) with a block size of $P$. The averaged signal is then 


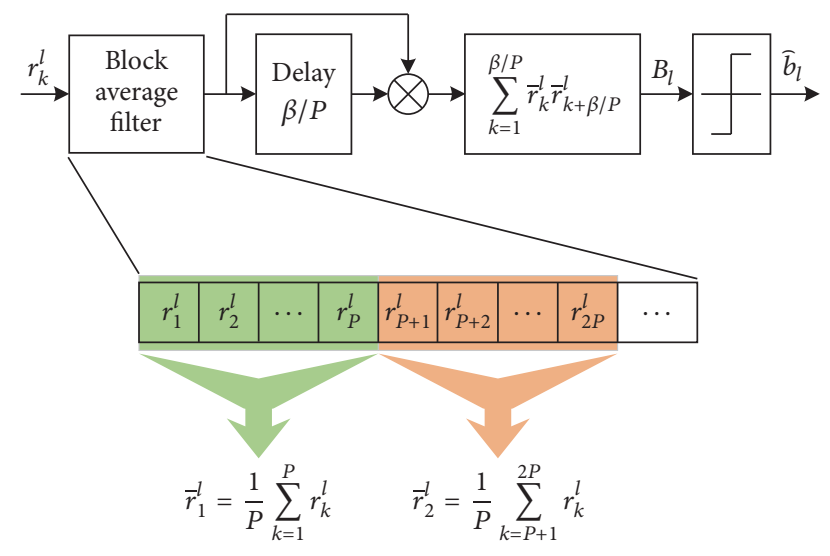

FIGURE 3: Receiver's structure and operation of the NR-DCSK system.

correlated with its replica that is time delayed by a factor of $\beta / P$. Thereafter, the resultant correlated signal is summed over $\beta / P$ samples and passed through a threshold detector to recover the transmitted information bit. The BAF is used to reduce the power of the AWGN, from which the noisy performance enhancement of the NR-DCSK can be achieved. A detailed illustration of the receiver is given in Figure 3, in which the output of the BAF would be given as

$$
\begin{aligned}
\bar{r}_{k}^{l} & =\frac{1}{P} \sum_{p=(k-1) P+1}^{k P} r_{p}^{l} \\
& =s_{k}^{l}+\frac{1}{P} \sum_{p=(k-1) P+1}^{k P} j_{p}^{l}+\frac{1}{P} \sum_{p=(k-1) P+1}^{k P} n_{p}^{l} .
\end{aligned}
$$

In addition, the decision variable at the input of the threshold detector can be written as

$$
\begin{aligned}
B_{l}= & \sum_{k=1}^{\beta / P}\left(x_{k}^{l}+\frac{1}{P} \sum_{p=(k-1) P+1}^{k P} j_{p}^{l}+\frac{1}{P} \sum_{p=(k-1) P+1}^{k P} n_{p}^{l}\right) \\
& \cdot\left(b_{l} x_{k}^{l}+\frac{1}{P} \sum_{p=(k-1) P+1}^{k P} j_{p+\beta}^{l}+\frac{1}{P} \sum_{p=(k-1) P+1}^{k P} n_{p+\beta}^{l}\right) .
\end{aligned}
$$

\section{Jamming Models}

In this section, we shall present several jamming types that are commonly encountered in military systems. These common jamming types include BBJ, PBJ, TJ, and SWJ.

3.1. Broadband Jamming [13]. A broadband jammer places jamming energy across the entire frequency bandwidth used by the target communication system. The BBJ essentially raises the background noise level at the receiver, creating a higher noise environment, which makes it more difficult for the target communication system to operate. At the very least it decreases the range over which the target communication system is effective. Since BBJ generates signals that are similar to broadband background noise, it is commonly modeled as a zero-mean Gaussian random variable with variance (power) $P_{j}$.
3.2. Partial-Time Jamming [14]. A partial-time jammer turns on and off periodically according to the jamming factor $\rho=$ $T_{\text {on }} /\left(T_{\text {on }}+T_{\text {off }}\right)$, where $T_{\text {on }}$ and $T_{\text {off }}$ denote the periods in which the jammer is on and off, respectively. In addition, the summation of $T_{\text {on }}$ and $T_{\text {off }}$ is called the jamming duty cycle. When the jammer is on, it is commonly assumed to emit broadband signals, that is, Gaussian noise, with power $P_{j} / \rho$, where $P_{j}$ is the average jamming power. It means that the jammer turns on and off periodically to focus more power on degrading the communication of the target system. It is noteworthy that the $\mathrm{BBJ}$ is a special case of the PTJ when $\rho=1$.

3.3. Tone Jamming [15]. In TJ, one or more jammer tones are strategically placed in the target signals' spectrum. TJ includes STJ and multiple-tone jamming (MTJ). STJ places a single-tone where it is needed, while MTJ equally distributes the jamming power among several tones. Mathematically, the TJ can be expressed as follows:

$$
J_{t j}(t)=\sum_{m=1}^{M} \sqrt{\frac{2 P_{j}}{M}} \sin \left(2 \pi f_{m} t+\theta_{m}\right),
$$

where $M, P_{j}, f_{m}$, and $\theta_{m}$ are the number of jamming tones, total jamming power, and the frequency and the phase of the $m$ th jamming tone, respectively. In addition, it should be noticed that $f_{m}$ here is the baseband frequency of the $m$ th jamming tone, which is different from the passband counterpart; that is, $f_{m}+f_{c}$ where $f_{c}$ denotes the carrier frequency of the transmitted chaotic signals.

3.4. Sweep Jamming [16]. A sweep jammer rapidly sweeps a narrow-band jamming signal over a wide frequency band. The SWJ can be characterized by its starting frequency $f_{\text {start }}$, stopping frequency $f_{\text {stop }}$, sweep rate $\Delta f$, and sweep time $T_{\text {sw }}$. In addition, there are several variants of the SWJ depending on narrow-band signals being used and sweep methods that specify the evolution of the instantaneous sweep frequency. Generally, narrow-band signals used to generate the SWJ include sinusoidal, triangular, and rectangular pulses. Moreover, sweep methods contain linear, quadratic, and logarithmic. Among various aforementioned options, the one based on sinusoidal signals and linear sweep method is commonly used and modeled as follows:

$$
J_{\text {sw }}(t)=\sqrt{2 P_{j}} \sin \left(2 \pi f_{\text {start }} t+\pi \Delta f t^{2}+\theta_{\text {sw }}\right),
$$

where $\Delta f=\left(f_{\text {stop }}-f_{\text {start }}\right) / T_{\text {sw }}$ and $\theta_{\text {sw }}$ denotes the initial phase of the sweep jamming signals. Similar to the case of the TJ, $f_{\text {start }}$ and $f_{\text {stop }}$ here are all the baseband frequencies.

\section{Antijamming Performance Analysis}

In this section, we analytically analyse the BER of the NRDCSK system under the PTJ environment. In addition, since the $\mathrm{BBJ}$ is a special case of the PTJ, the system BER under the BBJ environment can be straightforwardly obtained from that under the PTJ. The system performances under the other 
types of jamming will be evaluated via Monte-Carlo numerical simulations in the next section due to mathematical intractability.

The decision variable presented in (5) can be further expanded as follows:

$$
\begin{aligned}
& B_{l}=\underbrace{\sum_{k=1}^{\beta / P} b_{l}\left(x_{k}^{l}\right)^{2}}_{U}+\underbrace{\sum_{k=1}^{\beta / P} \frac{x_{k}^{l}}{P} \sum_{p=(k-1) P+1}^{k P} j_{p+\beta}^{l}}_{I_{1}} \\
& +\underbrace{\sum_{k=1}^{\beta / P} \frac{b_{l} x_{k}^{l}}{P} \sum_{p=(k-1) P+1}^{k P} j_{p}^{l}}_{I_{2}} \\
& +\underbrace{\sum_{k=1}^{\beta / P} \frac{1}{P^{2}} \sum_{p=(k-1) P+1}^{k P} j_{p}^{l} \sum_{p=(k-1) P+1}^{k P} j_{p+\beta}^{l}}_{I_{3}} \\
& +\underbrace{\sum_{k=1}^{\beta / P} \frac{x_{k}^{l}}{P} \sum_{p=(k-1) P+1}^{k P} n_{p+\beta}^{l}}_{N_{1}}+\underbrace{\sum_{k=1}^{\beta / P} \frac{b_{l} x_{k}^{l}}{P} \sum_{p=(k-1) P+1}^{k P} n_{p}^{l}}_{N_{2}} \\
& +\underbrace{\sum_{k=1}^{\beta / P} \frac{1}{P^{2}} \sum_{p=(k-1) P+1}^{k P} j_{p}^{l} \sum_{p=(k-1) P+1}^{k P} n_{p+\beta}^{l}}_{N_{3}} \\
& +\underbrace{\sum_{k=1}^{\beta / P} \frac{1}{P^{2}} \sum_{p=(k-1) P+1}^{k P} n_{p}^{l} \sum_{p=(k-1) P+1}^{k P} j_{p+\beta}^{l}}_{N_{4}} \\
& +\underbrace{\sum_{k=1}^{\beta / P} \frac{1}{P^{2}} \sum_{p=(k-1) P+1}^{k P} n_{p}^{l} \sum_{p=(k-1) P+1}^{k P} n_{p+\beta}^{l}}_{N_{5}},
\end{aligned}
$$

where $U, I_{i}$, and $N_{j}$ represent the desired, interference, and noise components. It is noteworthy that $U, I_{i}$, and $N_{j}$ are independent because the chaotic signal, jamming signal, and AWGN are independent. In addition, at any given time, the chaotic signal is independent of its time-delayed version. Under the PTJ condition, the jamming signal $j_{k}$ is modeled as a zero-mean Gaussian random variable with variance (power) $P_{j} / \rho$, where $P_{j}$ is the average jamming power. In addition, the power of the AWGN $n_{k}$ is assumed to be $N_{0} / 2$.

Following the central limit theorem, the decision variable $B_{l}$ can be assumed to follow the Gaussian distribution, and thus the error probability of transmitting the bit $b_{l}$ can be calculated as follows:

$$
\begin{aligned}
\mathrm{BER}_{l}= & \frac{1}{2} \operatorname{Pr}\left[B_{l}<0 \mid b_{l}=+1\right] \\
& +\frac{1}{2} \operatorname{Pr}\left[B_{l}>0 \mid b_{l}=-1\right] \\
= & \frac{1}{2} \operatorname{erfc}\left[\left(\frac{2 \operatorname{var}\left[B_{l}\right]}{\left(E\left[B_{l}\right]\right)^{2}}\right)^{-1 / 2}\right],
\end{aligned}
$$

where $\operatorname{erfc}(\cdot), \operatorname{var}[\cdot]$, and $E[\cdot]$ are the complementary error function, the variance, and the mean operations, respectively. Equation (9) indicates that, to obtain $\mathrm{BER}_{l}$, the mean and variance of $B_{l}$ are required. Firstly, the mean of $B_{l}$ can be readily derived as follows:

$$
E\left[B_{l}\right]=E[U]=b_{l} E\left[\sum_{k=1}^{\beta / P}\left(x_{k}^{l}\right)^{2}\right]=b_{l} E_{P} .
$$

To derive the variance of $B_{l}$, it is necessary to consider whether the jammer is on or off during the transmission time of the bit $b_{l}$. Let us first consider the case that the jammer is on. Under this condition, the bit $b_{l}$ can be assumed to be jammed with a probability of $\rho$. With a typical assumption of a high spreading factor, the bit energy $E_{b}=2 P \sum_{k=1}^{\beta / P} E\left[\left(x_{k}^{l}\right)^{2}\right]=$ $2 P E_{P}$ can be considered to be constant [8-10]. In addition, we can readily show that the average of $P$ independent and identically distributed (i.i.d.) samples of a zero-mean Gaussian variable with variance of $X$ is still a zero-mean Gaussian random variable with a reduced variance of $X / P$. Based on this result, we can derive the following results:

$$
\operatorname{var}\left[B_{l}\right]=\sum_{i=1}^{3} \operatorname{var}\left[I_{i}\right]+\sum_{j=1}^{5} \operatorname{var}\left[N_{j}\right],
$$

where $\operatorname{var}\left[I_{i}\right]$ and $\operatorname{var}\left[N_{j}\right]$ are given by

$$
\begin{aligned}
\operatorname{var}\left[I_{1}\right] & =\frac{E_{P} P_{j}}{\rho P}=\operatorname{var}\left[I_{2}\right], \\
\operatorname{var}\left[I_{3}\right] & =\frac{\beta P_{j}^{2}}{\rho^{2} P^{3}}, \\
\operatorname{var}\left[N_{1}\right] & =\frac{E_{P} N_{0}}{2 P}=\operatorname{var}\left[N_{2}\right], \\
\operatorname{var}\left[N_{3}\right] & =\frac{\beta P_{j} N_{0}}{2 \rho P^{3}}=\operatorname{var}\left[N_{4}\right], \\
\operatorname{var}\left[N_{5}\right] & =\frac{\beta N_{0}^{2}}{4 P^{3}} .
\end{aligned}
$$

Plugging all the results given in (10)-(12) into (9) yields the following error probability of transmitting the bit $b_{l}$ while the PTJ jammer is on:

$$
\begin{aligned}
& \mathrm{BER}_{l}^{\text {on }}=\frac{1}{2} \operatorname{erfc}\left[\left(\frac{4 P_{j} / \rho+2 N_{0}}{P E_{P}}\right.\right. \\
& \left.\left.+\beta \frac{2 P_{j} N_{0} / \rho+2 P_{j}^{2} / \rho^{2}+N_{0}^{2} / 2}{P^{3} E_{P}^{2}}\right)^{-1 / 2}\right] .
\end{aligned}
$$

On the other hand, when the partial-time jammer is off during the transmission time of the bit $b_{l}$, we can readily obtain the corresponding error probability by setting $P_{j}=0$ in (13). That is,

$$
\mathrm{BER}_{l}^{\text {off }}=\frac{1}{2} \operatorname{erfc}\left[\left(\frac{2 N_{0}}{P E_{P}}+\beta \frac{N_{0}^{2} / 2}{P^{3} E_{P}^{2}}\right)^{-1 / 2}\right] .
$$


Then, a weighted summation of (13) and (14), whose weighting factors are the probabilities that the bit $b_{l}$ is jammed and unjammed, produces

$$
\begin{aligned}
& \mathrm{BER}_{l}=\frac{\rho}{2} \operatorname{erfc}\left[\left(\frac{4 P_{j} / \rho+2 N_{0}}{P E_{P}}\right.\right. \\
& \left.\left.+\beta \frac{2 P_{j} N_{0} / \rho+2 P_{j}^{2} / \rho^{2}+N_{0}^{2} / 2}{P^{3} E_{P}^{2}}\right)^{-1 / 2}\right]+\frac{1-\rho}{2} \\
& \cdot \operatorname{erfc}\left[\left(\frac{2 N_{0}}{P E_{P}}+\beta \frac{N_{0}^{2} / 2}{P^{3} E_{P}^{2}}\right)^{-1 / 2}\right] .
\end{aligned}
$$

We observe that the above $\mathrm{BER}_{l}$ does not depend on the bit index $l$, and thus the system BER under the PTJ environment can be expressed as follows:

$$
\begin{aligned}
& \mathrm{BER}_{\mathrm{PTJ}}=\frac{\rho}{2} \operatorname{erfc}\left[\left(8 \frac{P_{j} / \rho+N_{0} / 2}{E_{b}}\right.\right. \\
& \left.\left.+8 \beta \frac{\left(P_{j} / \rho+N_{0} / 2\right)^{2}}{P E_{b}^{2}}\right)^{-1 / 2}\right]+\frac{1-\rho}{2} \operatorname{erfc}\left[\left(\frac{4 N_{0}}{E_{b}}\right.\right. \\
& \left.\left.+\frac{2 \beta N_{0}^{2}}{P E_{b}^{2}}\right)^{-1 / 2}\right] .
\end{aligned}
$$

Under the special case of the BBJ environment, the system BER can also be readily obtained from (16) by setting $\rho=1$. Particularly,

$$
\begin{aligned}
& \mathrm{BER}_{\mathrm{BBJ}} \\
& =\frac{1}{2} \operatorname{erfc}\left[\left(8 \frac{P_{j}+N_{0} / 2}{E_{b}}+8 \beta \frac{\left(P_{j}+N_{0} / 2\right)^{2}}{P E_{b}^{2}}\right)^{-1 / 2}\right] .
\end{aligned}
$$

It is observed from (16) and (17) that as $P$ increases, the BERs are reduced and tend to the lower bound given by

$$
\begin{aligned}
\operatorname{BER}_{\mathrm{PTJ}}^{\text {lower }}= & \frac{\rho}{2} \operatorname{erfc}\left[\left(\frac{8}{E_{b}}\left(\frac{P_{j}}{\rho}+\frac{N_{0}}{2}\right)\right)^{-1 / 2}\right] \\
& +\frac{1-\rho}{2} \operatorname{erfc}\left[\left(\frac{4 N_{0}}{E_{b}}\right)^{-1 / 2}\right] .
\end{aligned}
$$

Taking a closer look at (16) and (18), we can observe that when $P_{j}$ is large enough, the partial-time jammer should select $\rho=$ 1 to optimally degrade the performance of the target system. On the other hand, when $P_{j}$ is small, it is obvious that $\rho$ should be chosen close to zero to increase the instantaneous jamming power, and thus it further deteriorates the performance of the target system.

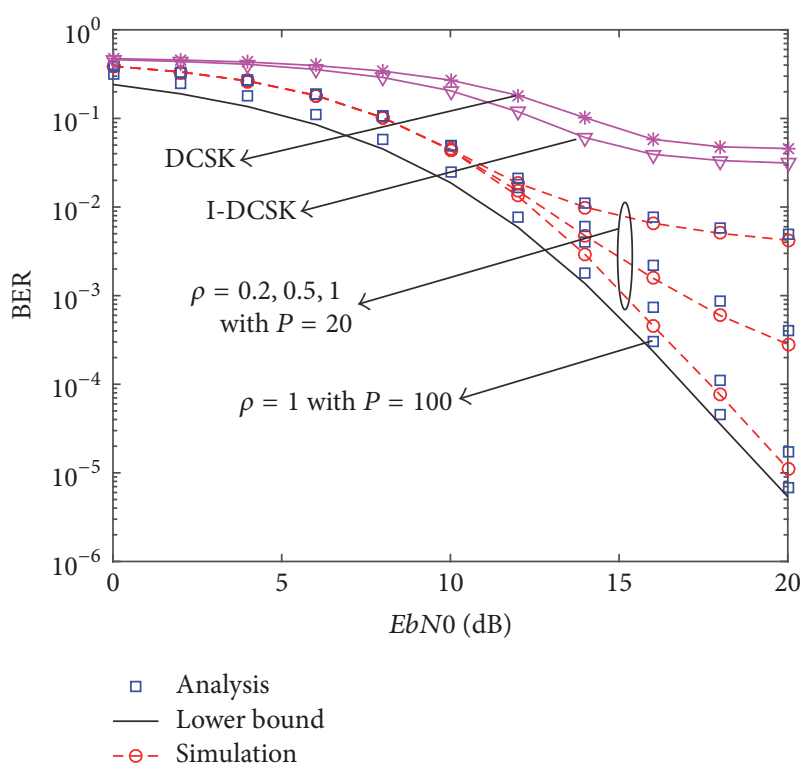

FIGURE 4: The system BERs under the PTJ and the BBJ environments with JSR $=P_{j} / P_{s}=5 \mathrm{~dB}$.

\section{Simulation Results}

In this section, we shall provide several representative simulation results to (i) verify our analysis on the system BERs under the BBJ and the PTJ environments and (ii) illustrate the effects of the TJ and the SWJ on the system performance.

In Figure 4, we simulate the BER of the NR-DCSK system versus $E_{b} / N_{0}$ under the $\mathrm{PTJ}$ and the $\mathrm{BBJ}$ environments with JSR $=P_{j} / P_{s}=5 \mathrm{~dB}$. For a comparison purpose, the BERs of the DCSK and the I-DCSK systems are also plotted. The figure first shows that the analysis curves follow the simulated ones closely, which validates our analysis. Secondly, we observe that with JSR $=5 \mathrm{~dB}$ the system BER is enhanced as the jamming factor $\rho$ increases. In other words, from the jammer's perspective, it is better for the jammer to be active with a short duration and transmit with a higher power to more severely degrade the target system performance. Thirdly, the figure confirms that as $P$ enlarges, the system BER improves and tends to the lower bound given in (18). Lastly, the figure illustrates that the NR-DCSK system significantly outperforms the DCSK and the I-DCSK counterparts, which highlights the advantage of the NR-DCSK system as well as the importance of the parameter $P$.

To further illustrate the effects of the jamming factor $\rho$ of the PTJ on the system performance, we present the system BER versus $\rho$ and $P_{j}$ in Figure 5. We observe the trend that as $P_{j}$ is small, the optimal value of $\rho$ (from the jammer point of view) is close to zero. In addition, as $P_{j}$ increases, the optimal value of $\rho$ also increases and approaches one. This observation is understandable because when the jamming power is small, if the jamming is active all the time, the jamming power density will be very small, and thus the jamming effect on the system performance could be negligible. Therefore, it is optimal for the jammer to turn on for a short period of time and transmit with a much higher 


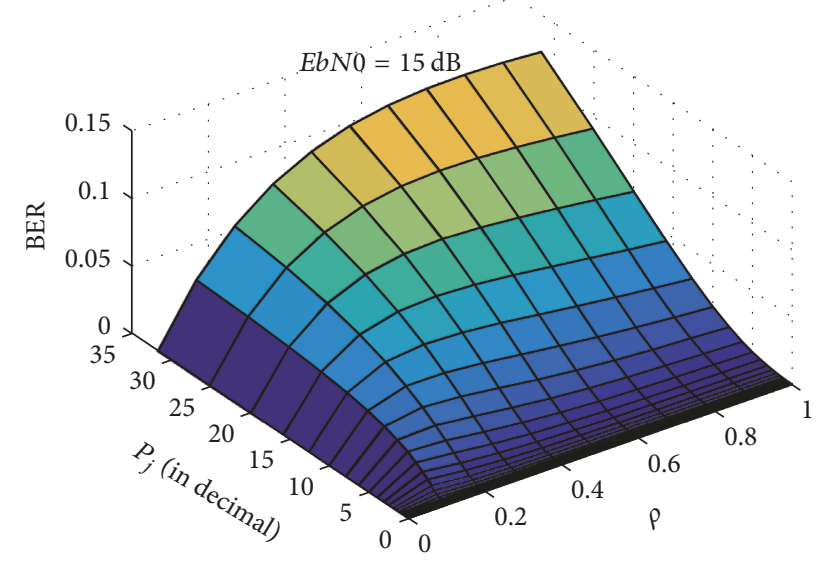

FIgure 5: The system BERs versus $\rho$ and $P_{j}$ with $P=20$ and $E b N 0=$ $15 \mathrm{~dB}$.

power. On the other hand, when the jamming power is larger than a certain value, the composite jamming and AWGN power is comparable with (or even larger than) that of the targeted signal, and thus it is unnecessary to further increase the jamming power. As a result, the jammer should turn on all the time to optimally degrade the target system performance. It is noteworthy that, given $E_{b} / N_{0}, \beta, P$, and $P_{j}$, the optimal value of $\rho$ can be easily derived by numerically solving the optimization problem, whose objective function is (16), with mathematical programs such as Matlab or Mathematica, that is, with $E_{b} / N_{0}=15 \mathrm{~dB}, \beta=200, P=20$, and $P_{j}=10 \mathrm{~dB}$, the optimal value of $\rho$ is found to be 0.24576 .

We now turn our interest to the effect of the TJ on the performance of the NR-DCSK system. In the simulation, we use the following discrete baseband model of the TJ signals [11]:

$$
j_{k}=\sum_{m=1}^{M} \sqrt{\frac{2 P_{j}}{M}} \sin \left(\pi k \frac{F_{m}}{\beta}+\theta_{m}\right),
$$

where $F_{m}=f_{m} T_{b}$ is the normalized jamming frequency of the $m$ th tone and $T_{b}$ denotes the bit duration. Here, $\theta_{m}$ is modeled as an arbitrary constant angle selected from $[-\pi, \pi]$. In addition, we simulate the system BER under single-tone, 3-tone, and 5-tone cases. It is shown in Figure 6 that the STJ causes the most significant performance degradation and increasing the number of jamming tones actually enhances the performance of the target system. Moreover, for the STJ case, increasing $P$ can improve the system performance. The reason is that, with a larger value of $P$, the receiver can further reduce the composite power of jamming and AWGN (by using the BAF), and thus the SINR at the receiver is improved, from which the system BER is enhanced. Finally, it is observed that as JSR is reduced, the system BER decreases, as expected.

The effects of the SWJ on the performance of the NRDCSK system will now be presented. According to [17], we

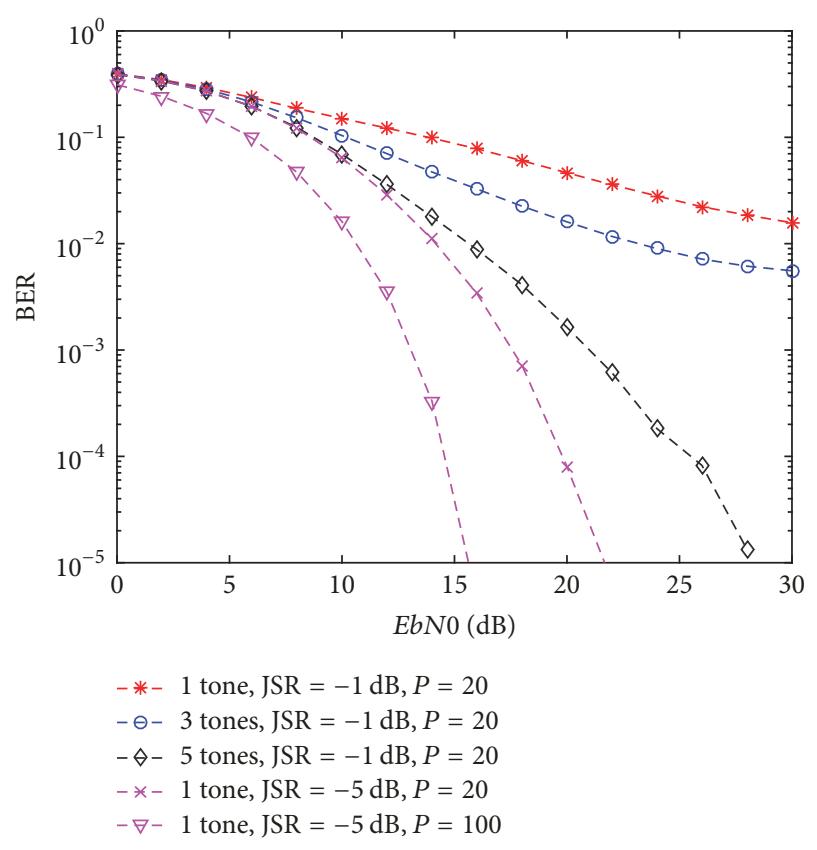

FIGURE 6: The system BERs versus EbN0 under the TJ environment.

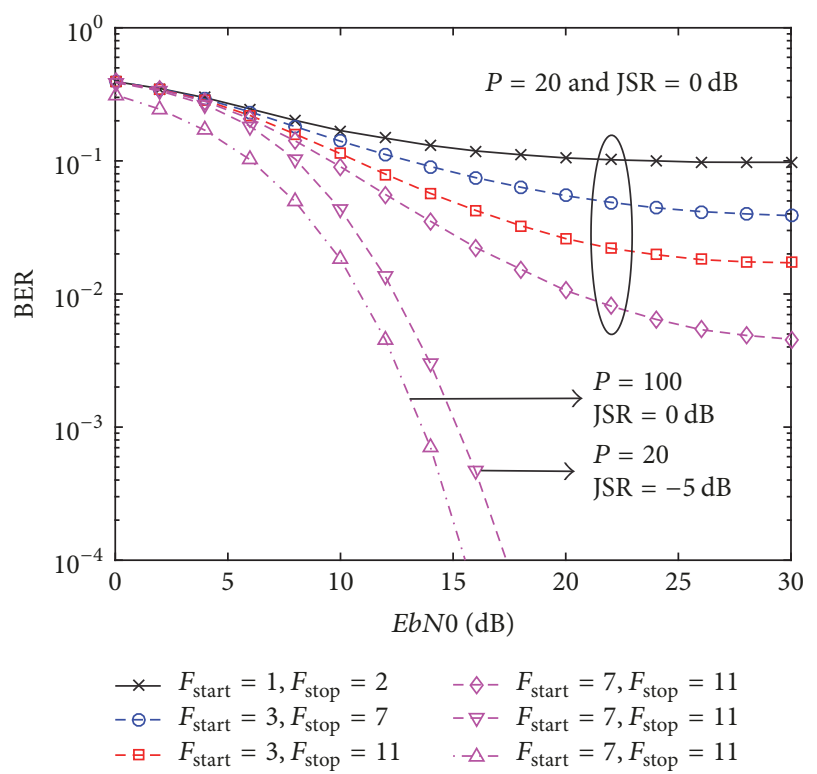

FIgURE 7: The system BERs versus EbN0 under the SWJ environment.

can use the following discrete baseband model of the SWJ signals in our simulation:

$$
j_{k}=\sqrt{2 P_{j}} \sin \left(\pi \frac{k F_{\text {start }}}{\beta}+\pi \frac{k^{2} \Delta F}{4 \beta^{2}}+\theta_{\text {sw }}\right),
$$

where $F_{\text {start }}=f_{\text {start }} T_{b}$ and $\Delta F=\Delta f T_{b}^{2}$. In Figure 7, we simulate the system BER versus $E b N 0$ with the condition that the sweep time equals the bit time, from which $\Delta F=$ $F_{\text {stop }}-F_{\text {start }}$, where $F_{\text {stop }}=f_{\text {stop }} T_{b}$. The figure shows that the sweep frequencies and the sweep bandwidth have a great impact on the system performance. Particularly, the closer, 


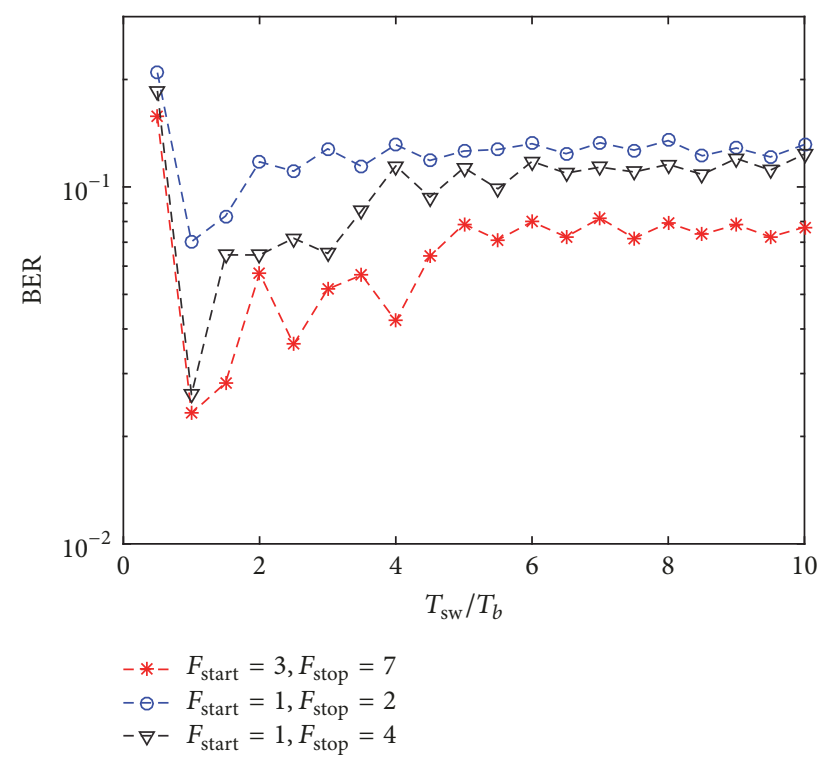

Figure 8: The system BERs versus $T_{\text {sw }}$ under the SWJ environment with $P=20$ and JSR $=0 \mathrm{~dB}$.

to the carrier frequency of the chaotic signals, the starting frequency of the sweep jammer is and the smaller the sweep bandwidth is, the more significant the system performance is degraded. In addition, as expected, with a larger $P$ or with a smaller JSR, the system performance is enhanced.

In Figure 8, we present the system BER versus the ratio of the sweep time and the bit duration with $P=20$ and JSR $=0 \mathrm{~dB}$. It can be seen that the most effective sweep time is half of the bit duration. The reason is that when $T_{\mathrm{sw}}=T_{b} / 2$, the reference and the information bearing sequences of any bit suffer from the same SWJ signals, which increases the correlation between the two sequences regardless of the bit value. Consequently, the decoding performance is degraded. In addition, the figure shows that when the sweep time is much larger than the bit duration, its effect on the system performance is negligible.

The effects of $\beta$ on the system performance are illustrated in Figure 9. We observe that, under the SWJ, the smaller $\beta$ the better the system BER. In addition, as $\beta$ increases, the system BER quickly converges to a saturation value. On the other hand, optimal values of $\beta$ under the PTJ and the STJ are similar and depend on the jamming parameters as well as $P$. As $P$ increases, the optimal values of $\beta$ also increase. When $\beta$ is less than the optimal values, increasing $\beta$ enhances the system BERs, while the converse holds when $\beta$ enlarges from the optimal values. Moreover, under the STJ, as $\beta$ enlarges from the optimal value, the system BER converges to that caused by the SWJ counterpart. It is also seen that the larger $P$ the better the system BERs. This trend is also observed from a figure showing the system BER versus $P$, which is not presented here for brevity.

\section{Conclusion}

In this work, we considered the NR-DCSK system under the BBJ, PTJ, TJ, and SWJ environments. We analytically derived

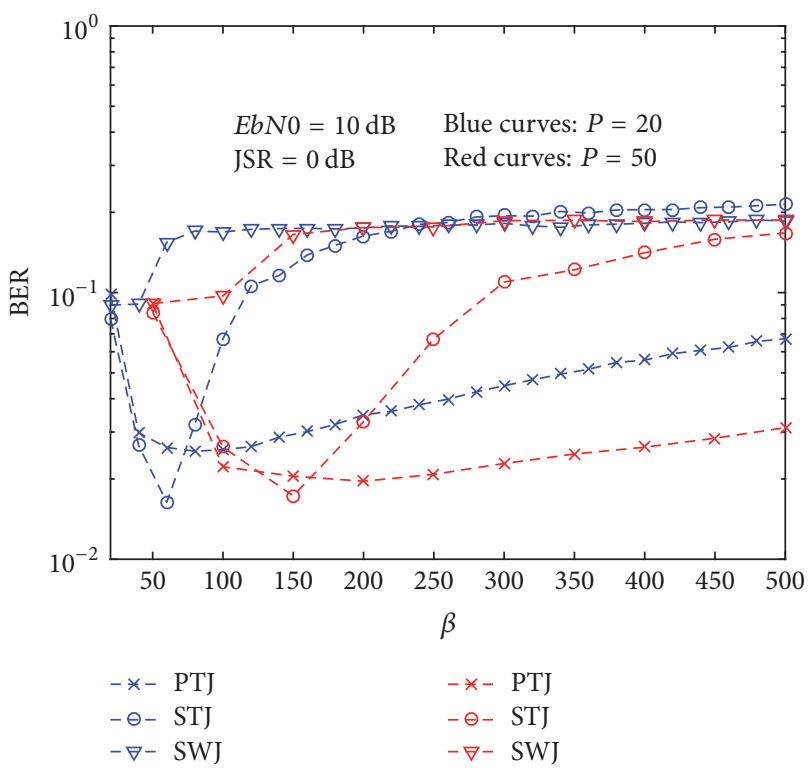

FIGURE 9: The system BERs versus $\beta E b N 0=10$ and $\mathrm{JSR}=0 \mathrm{~dB}$.

closed-form expressions of the system BERs under the BBJ and the PTJ environments, from which we revealed that in general increasing $P$ can enhance the system antijamming performance. In addition, we showed that the optimal value of the jamming factor of the PTJ tends to zero when the jamming power is small; however, it increases and approaches one as the jamming power increases. Moreover, via simulations, we demonstrated that the STJ is more efficient than the MTJ counterparts, from the tone jammers' point of view. Furthermore, we illustrated that under the SWJ environment, the system performance is more significantly degraded when the starting frequency of the sweep jammer is closer to the carrier frequency of the chaotic signals and the sweep bandwidth is small. Finally, we revealed that the most effective sweep time is half of the bit duration. Our results could be used as a guideline for systems' designers in turning existing systems' parameters to satisfy a certain quality-ofservice or designing novel and effective AJ communication systems.

\section{Conflicts of Interest}

The authors declare that they have no conflicts of interest.

\section{Acknowledgments}

The authors gratefully acknowledge the support from Electronic Warfare Research Center at Gwangju Institute of Science and Technology (GIST), originally funded by Defense Acquisition Program Administration (DAPA) and Agency for Defense Development (ADD).

\section{References}

[1] H. Yang, G.-P. Jiang, and J. Duan, "Phase-separated DCSK: a simple delay-component-free solution for chaotic communications," IEEE Transactions on Circuits and Systems II: Express Briefs, vol. 61, no. 12, pp. 967-971, 2014. 
[2] G. Kaddoum, "Wireless chaos-based communication systems: a comprehensive survey," IEEE Access, vol. 4, pp. 2621-2648, 2016.

[3] M. Sushchik, L. S. Tsimring, and A. R. Volkovskii, "Performance analysis of correlation-based communication schemes utilizing chaos," IEEE Transactions on Circuits and Systems I: Fundamental Theory and Applications, vol. 47, no. 12, pp. 1684-1691, 2000.

[4] M. Long, Y. Chen, and F. Peng, "Simple and accurate analysis of BER performance for DCSK chaotic communication," IEEE Communications Letters, vol. 15, no. 11, pp. 1175-1177, 2011.

[5] Y. Xia, C. K. Tse, and F. C. M. Lau, "Performance of differential chaos-shift-keying digital communication systems over a multipath fading channel with delay spread," IEEE Transactions on Circuits and Systems II: Express Briefs, vol. 51, no. 12, pp. 680684, 2004.

[6] H. Yang and G.-P. Jiang, "Reference-modulated DCSK: a novel chaotic communication scheme," IEEE Transactions on Circuits and Systems II: Express Briefs, vol. 60, no. 4, pp. 232-236, 2013.

[7] H. Yang and G.-P. Jiang, "High-efficiency differential-chaosshift-keying scheme for chaos-based noncoherent communication," IEEE Transactions on Circuits and Systems II: Express Briefs, vol. 59, no. 5, pp. 312-316, 2012.

[8] G. Kaddoum, E. Soujeri, C. Arcila, and K. Eshteiwi, "I-DCSK: an improved noncoherent communication system architecture," IEEE Transactions on Circuits and Systems II: Express Briefs, vol. 62, no. 9, pp. 901-905, 2015.

[9] G. Kaddoum, E. Soujeri, and Y. Nijsure, "Design of a short reference noncoherent chaos-based communication systems," IEEE Transactions on Communications, vol. 64, no. 2, pp. 680689, 2016.

[10] G. Kaddoum and E. Soujeri, "NR-DCSK: a noise reduction differential chaos shift keying system," IEEE Transactions on Circuits and Systems II: Express Briefs, vol. 63, no. 7, pp. 648652, 2016.

[11] F. C. M. Lau, M. Ye, C. K. Tse, and S. F. Hau, "Anti-jamming performance of chaotic digital communication systems," IEEE Transactions on Circuits and Systems I: Fundamental Theory and Applications, vol. 49, no. 10, pp. 1486-1494, 2002.

[12] F. C. M. Lau and C. K. Tse, "Performance of chaos-based digital communication systems in the presence of a pulsednoise jammer," Circuits, Systems and Signal Processing, vol. 23, no. 3, pp. 169-194, 2004.

[13] R. Poisel, Modern Communications Jamming Principles and Techniques, Artech House, 2nd edition, 2011.

[14] C. Xu, Y.-C. Liang, Y. L. Guan, and W. S. Leon, “Turbo product codes for mobile multimedia broadcasting with partial-time jamming," IEEE Transactions on Broadcasting, vol. 53, no. 1, pp. 256-261, 2007.

[15] W.-L. Mao, "Robust set-membership filtering techniques on GPS sensor jamming mitigation," IEEE Sensors Journal, vol. 17, no. 6, pp. 1810-1818, 2017.

[16] Y.-E. Chen, Y.-R. Chien, and H.-W. Tsao, "Chirp-like jamming mitigation for GPS receivers using wavelet-packet-transformassisted adaptive filters," in Proceedings of the International Computer Symposium (ICS '16), pp. 458-461, December 2016.

[17] S. G. Glisic, A. Mammela, V.-P. Kaasila, and M. D. Pajkovic, "Rejection of frequency sweeping signal in ds spread spectrum systems using complex adaptive filters," IEEE Transactions on Communications, vol. 43, no. 1, pp. 136-145, 1995. 


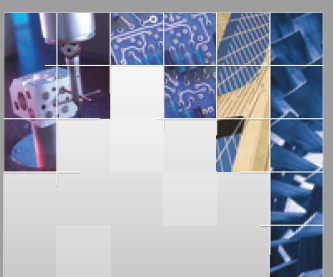

\section{Enfincering}
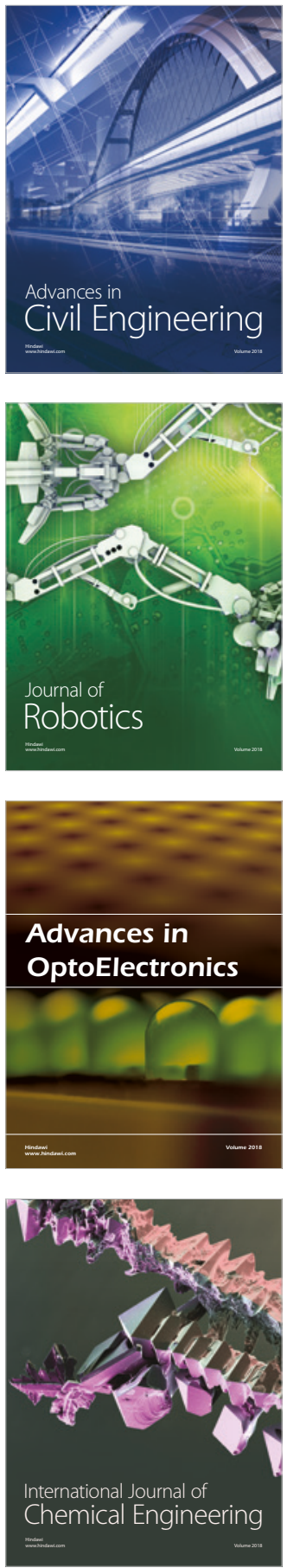

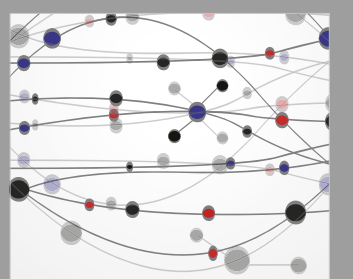

\section{Rotating \\ Machinery}

The Scientific World Journal

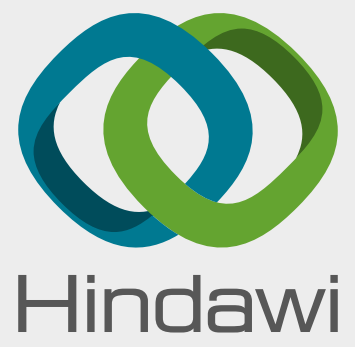

Submit your manuscripts at

www.hindawi.com
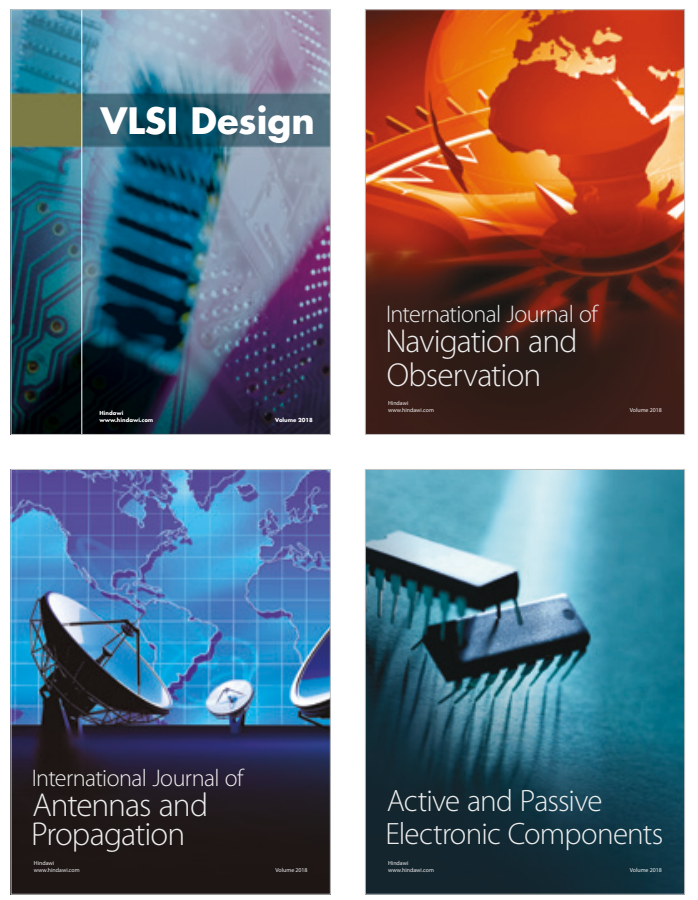
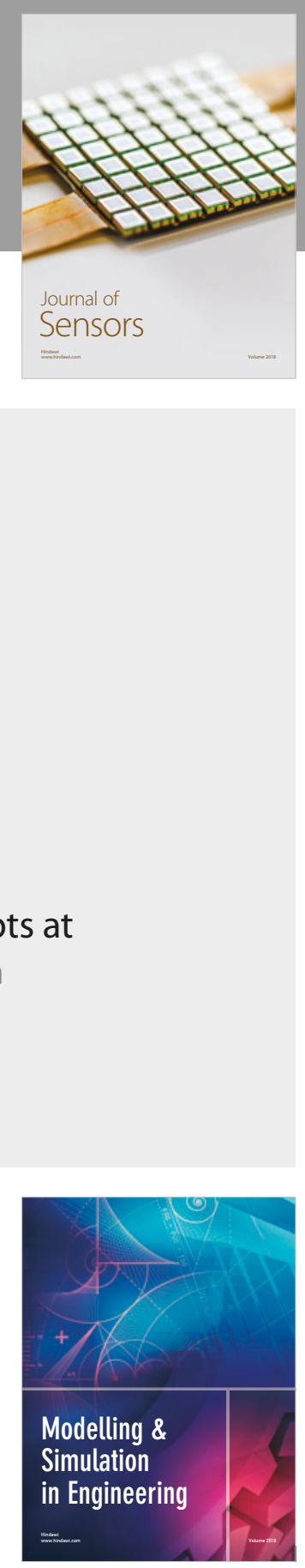

\section{Advances \\ Multimedia}
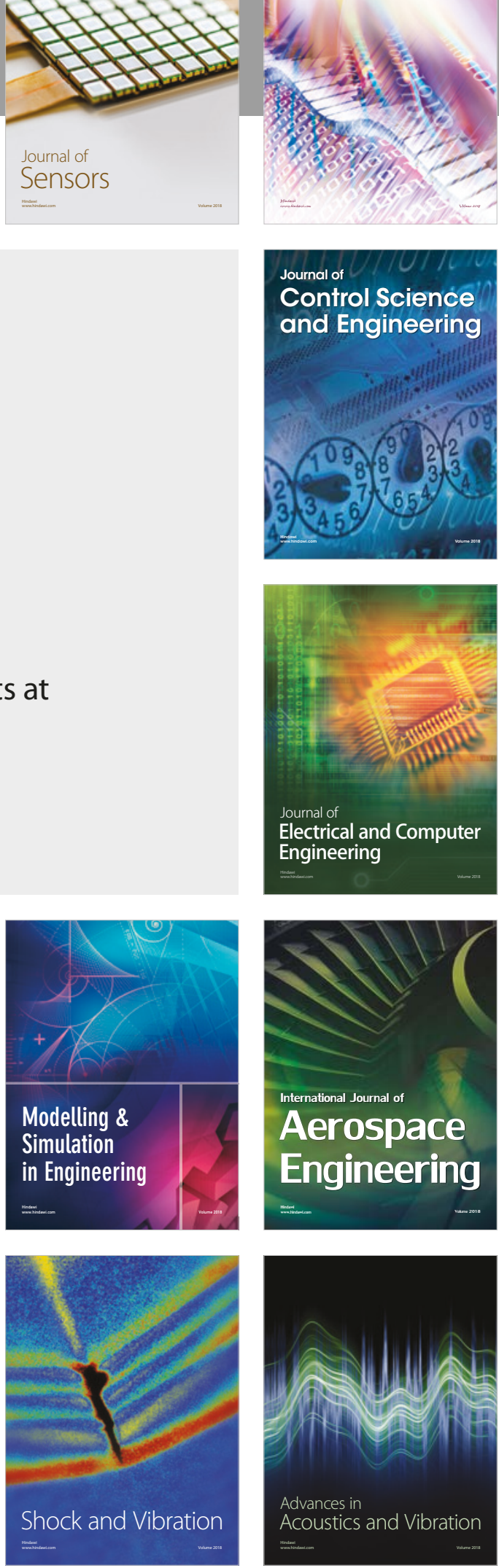